\title{
The Transition from Singly to Multiply-Charged Anomalous Cosmic Rays: Simulation and Interpretation of SAMPEX Observations
}

\author{
A.F. Barghouty ${ }^{1}$, J.R. Jokipii ${ }^{2}$, and R.A. Mewaldt ${ }^{1}$ \\ ${ }^{1}$ California Institute of Technology, Pasadena, CA 91125, USA \\ ${ }^{2}$ University of Arizona, Tuscon, AZ, 85721, USA
}

\begin{abstract}
Multiply-charged anomalous cosmic rays (ACRs) can arise when singly-charged ACR ions are stripped of one or more of their electrons during their acceleration via, e.g., the process of diffusive shock-drift acceleration at the solar-wind termination shock. Recent measurements of the charge states of ACR neon, oxygen, and nitrogen by SAMPEX at $1 \mathrm{AU}$ have shown that above $\approx 25 \mathrm{MeV} /$ nucleon these ions are multiply charged. In addition, SAMPEX observations have also established that the transition from mostly singly-charged to mostly multiply-charged ACRs (defined as the $50 \%$ point) occurs at a total kinetic energy of $\approx 350 \mathrm{MeV}$. Preliminary simulations for ACR oxygen based on a theory of multiply-charged ACRs were able to show a transition energy at $\approx 300 \mathrm{MeV}$. However, the simulated intensity distribution among the various charge states was inconsistent with observations. This paper reexamines the predictions of the theory in light of new SAMPEX ACR observations and recently developed and refined estimates of hydrogen-impact ionization cross sections. Based on simulations for multi-species ACR ions, we find that the transition energy is only weakly dependent on characteristic transport parameters, and that the new ionization rates distribute the intensity among the charge states in a manner consistent with observations. The calculated transition energy is in excellent agreement with the measured value.
\end{abstract}

\section{INTRODUCTION}

Unlike galactic cosmic rays (GCRs) or solar energetic particles ions (SEPs) observed at $1 \mathrm{AU}$, anomalous cosmic rays (ACRs) are expected to be predominantly singly-charged ions. According to the theory (1), ACRs are believed to originate as interstellar neutrals that penetrate the heliosphere before getting ionized -either by solar radiation or by charge-exchange collisions with solarwind protons - to become singly-charged pickup ions. These pickup ions are then convected by the solar wind to the solar-wind termination shock (SWTS) where they are accelerated up to tens of $\mathrm{MeV} /$ nucleon via, e.g., the process of diffusive shock-drift acceleration (2).

However, recent observational evidence from SAMPEX $(3,4)$ have shown that ACR neon, oxygen, and nitrogen above $\approx 25 \mathrm{MeV} /$ nucleon are multiply charged, with ionic charge states of 2,3, and higher. At energies lower than $\approx 20 \mathrm{MeV} /$ nucleon most of the observed ACRs are singly charged. SAMPEX observations (4) have further established that the transition from mostly singly-charged to mostly multiply-charged ACRs (defined as the 50\% point) occurs at a total kinetic energy of $\approx 350 \mathrm{MeV}$.

The predominance of multiply-charged ACRs at high energy has been interpreted as evidence that some ACR ions are stripped of one or more of their electrons during their acceleration at the SWTS $(3,6)$.

According to the theory of diffusive shock-drift acceleration $(5,6)$ the amount of energy an ACR ion gains in drifting along the SWTS (from the equator towards the poles during the 1990s) is proportional to its ionic charge $q$; energy gain $\approx 240 \times q \mathrm{MeV}$. As a result, multiplycharged ACRs are accelerated to higher energy per nucleon than the more abundant singly-charged ions. Thus, at energies $>240 \mathrm{MeV}$, the theory predicts that multiplycharged ACR ions will dominate.

Preliminary simulations based on a theory of multiplycharged ACRs (6) applied to ACR oxygen and using an older set of ionization cross sections (7), did show the presence of a transition energy around $300 \mathrm{MeV}$, above which the ACR oxygen is mostly multiply charged. However, the simulated intensity distribution among the charge states was skewed towards fully or almost fully stripped oxygen ions, in disagreement with SAMPEX observations (3).

The recent development $(8,9)$ of a refined set of ionization cross sections warrants a reexamination of the predictions of the theory. This paper focuses on the simula- 


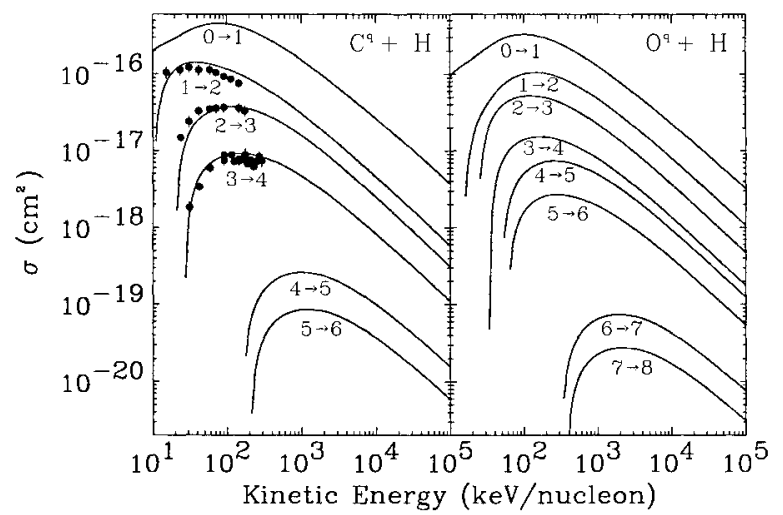

FIGURE 1. Estimated hydrogen-impact ionization cross sections for carbon and oxygen. Curves depict the cross sections $\sigma_{0 \rightarrow 1}$ (i.e., the differential cross section for the reaction $\left.\mathrm{X}^{0}+\mathrm{H} \rightarrow \mathrm{X}^{1}+\mathrm{H}^{*}+\mathrm{e}^{\prime}\right), \sigma_{1 \rightarrow 2}$, etc., and terminate as the $\mathrm{ki}-$ netic energy approaches the Born energy. [See (9) for reference on the experimental data for carbon.]

tion - using the new cross sections - and comparison to SAMPEX observations of ACR N, O, and Ne spectra at $1 \mathrm{AU}$, in particular, the observed transition from singly to multiply-charged ions.

\section{IONIZATION CROSS SECTIONS}

A new, theoretically-derived set of hydrogen-impact ionization cross sections applicable over a wide energy range has recently been developed $(8,9)$. This new crosssection set is derived using a formalism that is valid in the first Born approximation using measured (or estimated) electron-impact ionization cross sections (e.g., $(10,11,12))$. The formalism is based on a simple and elegant functional connecting the two sets of cross sections known as the Bates-Griffing relation. The Bates-Griffing relation is reformulated and a correction factor due to multiple transitions, i.e., sum over electrons' shells, is introduced. Fig. 1 shows a sample calculation using this new formalism for carbon and oxygen, where available data for carbon are also shown. Multi-electron removal is ignored in this calculation.

The previous simulations of multiply-charged ACRs (6) relied on ionization cross-section estimates based on oxygen-oxygen collisions (7). These estimates grossly overestimate the cross sections for multi-electron removal when applied to ion-hydrogen collisions. While data remain scarce for proton or hydrogen-impact ionization cross sections, multi-electron removal cross sections have been shown to be insignificant compared to single- electron removal in ion-electron collisions at energies relevant to ACR studies (e.g., $(13,14))$.

For hydrogen-impact collisions at such energies, multi-electron removal is also expected to be insignificant compared to single-electron removal. For example, the cross section for the two-electron removal process $\mathrm{p}+\mathrm{He} \rightarrow \mathrm{p}+\mathrm{He}^{+2}+2 \mathrm{e}$ at $1 \mathrm{MeV} /$ nucleon is found both experimentally (15) and theoretically (16) to be about 2.5 orders of magnitude smaller than that for the singleelectron removal process $\mathrm{p}+\mathrm{He} \rightarrow \mathrm{p}+\mathrm{He}^{+1}+1 \mathrm{e}$.

\section{SIMULATIONS}

The simulations presented here are produced using the acceleration and transport model described in (6). The model solves the time-dependent Parker transport equation in 2 dimensions with drift terms:

$$
\begin{aligned}
\frac{\partial f_{q}}{\partial t}= & \frac{\partial}{\partial x_{i}}\left(\kappa_{i j} \frac{\partial f_{q}}{\partial x_{j}}\right)-V_{w, i} \frac{\partial f_{q}}{\partial x_{i}} \\
& -V_{d, i} \frac{\partial f_{q}}{\partial x_{i}}+\frac{1}{3} \frac{\partial V_{w, i}}{\partial x_{i}} \frac{\partial f_{q}}{\partial \ln p} \\
& + \text { sources }_{q}-\operatorname{sinks}_{q},
\end{aligned}
$$

where $f_{q}(\vec{r}, p, t)$ is the distribution function of the ACR ion with charge $q$. From left to right, the RHS terms in the equation depict spatial diffusion, convection, drift, energy gains and losses, and particle sources and sinks due to ionization.

The salient transport parameters used for this study are tabulated in Table 1. In addition, the simulations are presented with and without taking differential solar rotation into account. The reader is referred to Ref. (17) in this volume for further discussion of the theory and simulations. Below we discuss the simulations as they pertain to the recent SAMPEX ACR observations (4).

\section{DISCUSSION}

Fig. 2 shows the calculated ACR oxygen spectra at 1 AU. Comparing Fig. 2 with Fig. 1 of Ref. (6) there are significant differences in the charge state distribution. In Fig. 1 of Ref. (6), using the older set of ionization cross sections (7), the calculated spectrum at higher energies is dominated by charges 7 and 8 , inconsistent with SAMPEX observations (3). In contrast, the new simulations show a summed spectrum that is dominated by charges 2 and 3 at higher energies. Although not shown in Fig. 2 for sake of clarity, the calculated contributions of charges 


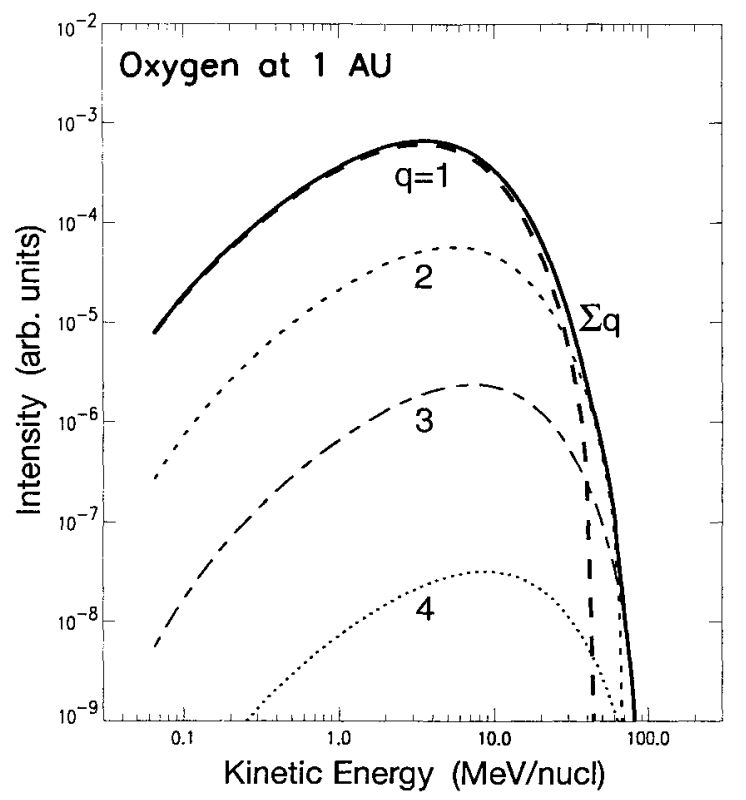

FIGURE 2. Simulated ACR oxygen spectra (in arbitrary units) at $1 \mathrm{AU}$. The top curve depicts the summed spectrum of charge states 1 through 8; while the second curve from the top depicts the spectrum for charge 1 ; third from the top charge 2 , etc. The spectra are simulated taking differential solar rotation into account.

7 and 8 to the total spectrum at both high and low energies are, consistent with observations, negligibly small. Simulated spectra for nitrogen and neon show very similar trends.

Because of the difficulty in resolving charge states higher than 3, Ref. (4) defines the transition energy as the energy that corresponds to the $50 \%$ point of the relative abundance of charge 1 to the sum of charges 1 through 3. Below that energy point the spectrum is dominated by singly-charged ACR ions and above it by multiplycharged ions. Using this definition of the transition energy, Fig. 3 shows the calculated ratio as a function of energy and compares it to the measured ratio (4). The measured transition energy for ACR oxygen is $22 \pm 2$ $\mathrm{MeV} /$ nucleon. In excellent agreement with observations, from Fig. 3, the calculated transition energy is $\approx 24$ $\mathrm{MeV} /$ nucleon. Again, although not shown, the same trends are seen for the calculated transition energies for nitrogen and neon.

In Fig. 4 we show the calculated transition energies for nitrogen, oxygen, neon as well as argon (although no measurement currently exists for argon) as a function of nuclear charge of the element. Multiply-charged ions can gain energy roughly in proportion to their ionic charge $x$ the SWTS electrostatic potential while being accelerated at the shock (18).

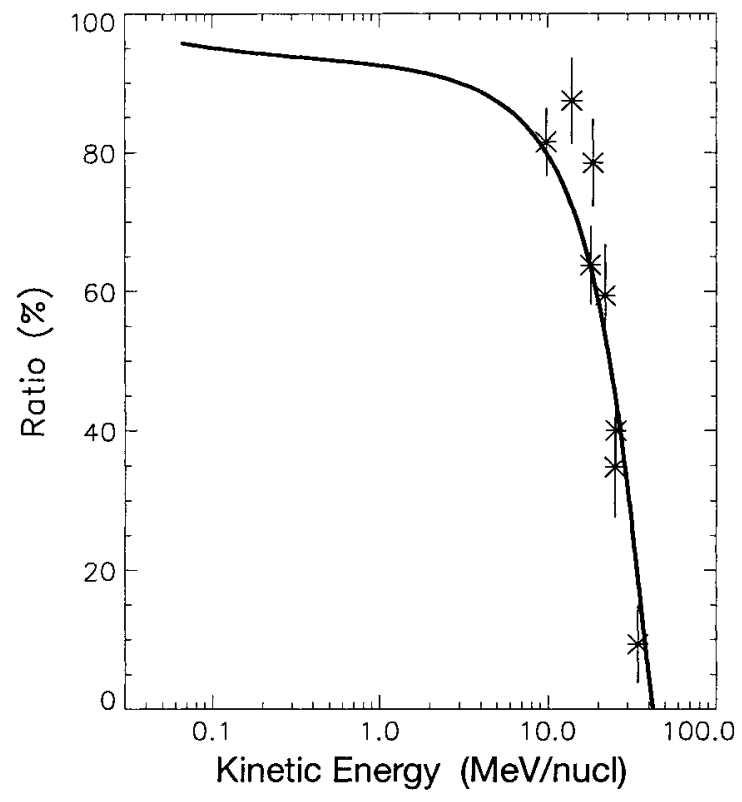

FIGURE 3. Calculated ratio of the singly-charged ACR oxygen to charges 1 through 3 as a function of energy. The $50 \%$ point is at $\approx 24 \mathrm{MeV} /$ nucleon. Data points are from Ref. (4).

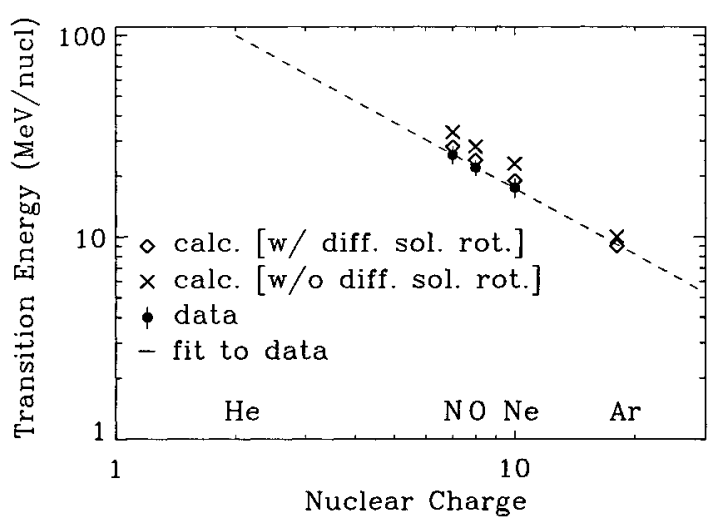

FIGURE 4. Calculated and measured 50\%-point transition energy as a function of nuclear charge demonstrating the effect of differential solar rotation. Data points are from Ref. (4).

Calculated transition energies with and without the effect of differential solar rotation are shown in Fig. 4 (simulated spectra are qualitatively unchanged). Although it is not easy to surmise directly from the simulation model, this effect can decrease the electrostatic potential between the equator and the poles so as to give a somewhat lower transition energies as evident from Fig. 4. For argon, the model predicts a transition energy of $\approx 9 \mathrm{MeV} /$ nucleon. 
Table 1. Simulation parameters:

\begin{tabular}{ll}
\hline Transport parameter & Value/Description \\
\hline Solar wind velocity & 350 (equator) $-700 \mathrm{~km} / \mathrm{s}$ (poles) \\
SWTS radius & $100 \mathrm{AU}$ \\
SWTS strength & 2.5 \\
Heliospheric boundary & $160 \mathrm{AU}$ \\
Neutral-H density & $0.16 \mathrm{~cm}^{-3}$ \\
Injection energy & $60 \mathrm{keV} /$ nucleon \\
Heliospheric $B$-field & Parker's+polar modification \\
& + flat current sheet \\
& $+q A>0$ conditions \\
Paral. diffusion coeff. & $\propto R^{1 / 2} \beta / B(\vec{r})$ \\
Perp. diffusion coeff. & $0.03 \times$ Paral. \\
\hline
\end{tabular}

${ }^{*} R$ is ion's rigidity, $\beta$ is its speed divided by the speed of light, and $B$ is the strength of the magnetic field at the heliospheric point $\vec{r}$

\section{CONCLUSIONS}

Using the new ionization cross-section estimates the simulated energy spectra of ACR oxygen, nitrogen, and neon are found to be distributed among the various charge states in a manner consistent with recent SAMPEX observations. The resulting transition energies from singly to multiply-charged ACR ions are in excellent agreement with the measured ones and are found to be only weakly dependent on characteristic heliospheric transport parameters. Differential solar rotation, however, is found to have a small but noticeable effect on the calculated transition energies.

The achieved level of agreement with observations has implications to particle acceleration models at the SWTS and quasi-perpendicular shocks in general. While the reader, again, is referred to Ref. (17) in this volume for further discussion of such implications, we conclude here that the agreement achieved in this study clearly supports the diffusive shock-drift acceleration model of ACRs. Also, the agreement underscores the critical role precise ionization cross sections play in this and other models of charged particle transport in the heliosphere.

\section{ACKNOWLEDGMENTS}

Work is supported by NSF grant no. 9810653 and NASA-JOVE NAG8-1208 (A.F.B.) and by NASA grants NAS5-30704 and NAG5-6912 at Caltech.

\section{REFERENCES}

1. L.A. Fisk, B. Koslovsky, and R. Ramaty, Astrophys. J. Lett. 190, L35 (1974).

2. M.E. Pesses, J.R. Jokipii, and D. Eichler, Astrophys. J. Lett. 246, L85 (1981).

3. R.A. Mewaldt et al., Astrophys. J. Lett. 466, L43 (1996).

4. B. Klecker et al., Space Sci. Rev. 83, 259 (1998).

5. J.R. Jokipii, in Physics of the Outer Heliosphere, eds. S. Grzedzielski and D.E. Page (Oxford: Pergamon), p. 169 (1990).

6. J.R. Jokipii, Astrophys. J. Lett. 466, L47 (1996).

7. W. Spjeldvik, Space Sci. Rev. 23, 499 (1979).

8. A.F. Barghouty, Proc. 26th Int. Cosmic-Ray Conf. (Salt Lake, Utah) 7, 555 (1999).

9. A.F. Barghouty, Phys. Rev. A61, 052702 (2000).

10. M. Arnaud and R. Rothenflug, Astron. \& Astrophys. (Suppl. Series) 60, 425 (1985).

11. M. Arnuad and J. Raymond, Astrophys. J. 398, 394 (1992).

12. V.P. Shevelko et al. 1983, Monthly Notices Royal Astron. Soc. 203, 45P (1983).

13. E. Krishnakumar and S.K. Srivastava, Int'l. J. Mass Spect. \& Ion Proc. 113, 1 (1992).

14. H. Deutch et al., Int'l. J. Mass Spect. 192, 1 (1999).

15. M.B. Shah and H.B. Gilbody, J. Phys. B 18, 899 (1985).

16. M.L. McKenzie and R.E. Olson, Phys. Rev. A 35, 2863 (1987).

17. J.R. Jokipii, this volume, (2000).

18. R.A. Mewaldt et al., Geophys. Res. Lett. 23, 617 (1996). 